\title{
BIOÉTICA E A ALOCAÇÃO DE RECURSOS NOS CUIDADOS PALIATIVOS DURANTE A PANDEMIA DE COVID-19: PERCEPÇÃO DE PROFISSIONAIS DE SAÚDE
}

\section{BIOETHICS AND THE ALLOCATION OF RESOURCES IN PALLIATIVE CARE DURING THE COVID-19 PANDEMIC: THE PERCEPTION OF HEALTH PROFESSIONALS}

\section{LA BIOÉTICA Y LA ASIGNACIÓN DE RECURSOS EN CUIDADOS PALIATIVOS DURANTE LA PANDEMIA DEL COVID-19 - PERCEPCIÓN DE LOS PROFESIONALES DE LA SALUD}

Priscila Kelly Silva Neto ${ }^{1}$, Juliana Dias Reis Pessalacia ${ }^{2}$, Elton Fogaça da Costa ${ }^{3}$, Paulo Roberto Haidamus de Oliveira Basto ${ }^{4}$, Luciana Regina Ferreira da Mata, Sandra Pinto ${ }^{5}$

\section{RESUMO}

Objetivo: Analisar a percepção de profissionais de saúde sobre as questões bioéticas na tomada de decisão acerca dos recursos escassos, no contexto dos Cuidados Paliativos (CP), durante a pandemia de COVID-19 no Brasil. Métodos: Estudo transversal, descritivo e de abordagem quantitativa, realizado por meio de um questionário on-line, contendo quatro afirmativas sobre os dilemas éticos para que os participantes pudessem concordar ou não com elas, totalizando um escore máximo de 20 pontos. Os dados foram tabulados e analisados, por meio de estatística descritiva, utilizando-se o software Statistical Package for Social Sciences (SPSS), versão 25.0. Resultados: Participaram 190 profissionais de saúde, 45,3\% (86) enfermeiros, 23,7\% (45) médicos, 10,5\% (20) dentistas e $12,6 \%$ (24) outros profissionais de saúde. Identificaram-se os seguintes percentuais de erros: restrição do acesso a Unidades de Terapia Intensiva (UTIs) (93 - 48,9\%), disponibilização de ventiladores (117 - 61,6\%), decisão de triagem para recursos escassos (158 - 83,2\%), interrupção do atendimento a pacientes crônicos e paliativos na pandemia (11 - 5,8\%). Conclusão: Os resultados demonstraram que os profissionais ainda apresentam conhecimento insuficiente sobre critérios de justiça e equidade na alocação de recursos escassos e que há necessidade de educação permanente no assunto voltado à temática.

Descritores: Bioética; Equidade em Saúde; Alocação de Recursos para a Atenção à Saúde; COVID-19; Cuidados Paliativos.

\begin{abstract}
Objective: To analyze the perception of health professionals about bioethical issues in decision-making processes about scarce resourcesfor Palliative Care (PC) during the COVID-19 pandemic in Brazil. Methods: This is a cross-sectional, descriptive study with a quantitative approach, carried out through an online questionnaire containing four statements about ethical dilemmas for participants to agree or disagree on, totaling a maximum score of 20 points. The data were tabulated and analyzed using descriptive statistics, on software Statistical Package for Social Sciences (SPSS), version 25.0. Results: 190 health professionals participated, $45.3 \%$ (86) nurses, $23.7 \%$ (45) doctors, $10.5 \%$ (20) dentists and $12.6 \%$ (24) other health professionals. The following percentages of errors were identified: restriction of access to Intensive Care Units (ICUs) (93 - 48.9\%), availability of ventilators (117 - 61.6\%), screening decision for scarce resources (158-83.2\%), interruption of care for chronic and palliative patients in the pandemic (11 - 5.8\%). Conclusion: The results demonstrate that the professionals still have insufficient knowledge about the criteria of justice and equity in the allocation of scarce resources and that there is a need for permanent education on the subject Descriptors: Bioethics; Health Equity; Resource Allocation for Health Care; COVID-19; Palliative Care.
\end{abstract}

\section{RESUMEN}

Objetivo: Analizar la percepción de los profesionales de la salud sobre temas bioéticos en la toma de decisiones sobre recursos escasos en el contexto de Cuidados Paliativos (CP) durante la pandemia de COVID-19 en Brasil. Métodos: Estudio descriptivo transversal con enfoque cuantitativo, realizado a través de un cuestionario online que contiene cuatro afirmaciones sobre dilemas éticos para que los participantes estén de acuerdo o en desacuerdo con ellos, totalizando una puntuación máxima de 20 puntos. Los datos fueron organizados y analizados mediante estadística descriptiva, utilizando el software Statistical Package for Social Sciences (SPSS), versión 25.0. Resultados: Participaron 190 profesionales de la salud, 45,3\% (86) enfermeras, 23,7\% (45) médicos, 10,5\% (20) odontólogos y 12,6\% (24) otros profesionales de la salud. Se identificaron los siguientes porcentajes de errores: restricción de acceso a Unidades de Cuidados Intensivos (UCI) (93-48,9\%), disponibilidad de ventiladores (117 - 61,6\%), decisión de selección de recursos escasos (158-83,2\%), interrupción de la atención de pacientes crónicos y paliativos en la pandemia (11 - 5,8\%). Conclusión: Los resultados demuestran que los profesionales aún tienen un conocimiento insuficiente sobre los criterios de justicia y equidad en la asignación de recursos escasos y que existe la necesidad de una educación permanente en la materia enfocada en el tema. Descriptores: Bioética; Equidad en Salud; Asignación de Recursos para la Atención Médica; COVID-19; Cuidados Paliativos.

${ }^{1}$ Mestranda em Enfermagem pela Universidade Federal de Mato Grosso do Sul. 2Pós doutorado em Enfermagem pela Escola de Enfermagem da Universidade de São Paulo, São Paulo. Docente da Universidade Federal de Mato Grosso do Sul. ${ }^{3}$ Doutor em Direito pela Universidade Federal de Santa Catarina. Docente da Universidade Federal de Mato Grosso do Sul. ${ }^{4}$ Doutor em Educação pela Pontifícia Universidade Católica de São Paulo. Docente da Universidade Federal de Mato Grosso do Sul. ${ }^{5}$ Doutora pelo programa Enfermagem Fundamental da Escola de Enfermagem de Ribeirão Preto da Universidade de São Paulo. Docente da Universidade Federal de Minas Gerais. ${ }^{6}$ Mestranda em Enfermagem pela Universidade Federal de Mato Grosso do Sul.

\section{Como citar este artigo:}

Neto PKS, Pessalácia JDR, Costa EF, et al. Bioética e a alocação de recursos nos cuidados paliativos durante a pandemia de Covid19: percepção de profissionais de saúde. Revista de Enfermagem do Centro-Oeste Mineiro. 2020;10:e4167. [Access Available in: DOI: http://doi.org/10.19175/recom.v10i0.4167 


\section{INTRODUÇÃO}

No Brasil, o Sistema Único de Saúde (SUS) garante acesso universal à saúde para qualquer cidadão do país. Contudo, em tempos de crise e com a escassez de recursos nos sistemas de saúde, os serviços passaram a enfrentar inúmeros desafios éticos pela falta de capacidade técnica e operacional, para lidar com um número inesperado de pacientes e com a tomada de decisão envolvendo a distribuição de recursos (humanos, materiais, equipamentos) de forma justa. Poucas doenças infecciosas novas ou emergentes apresentaram desafios éticos vitais de maneira tão rápida e dramática quanto o novo SARS-Cov-2, que causa o Corona Vírus Disease 2019 (COVID-19) $^{(1)}$.

Em situações de crise, como na atual pandemia de COVID-19, a ação humanitária tem um compromisso com as populações vulneráveis, incluindo as pessoas em Cuidados Paliativos (CP) que se encontram em condições de fim de vida. $A$ responsabilidade ética de fornecer $\mathrm{CP}$, em situações de crise humanitária, visa atender a um direito humano básico, incluindo-se o direito à dignidade e ao conforto no processo de adoecimento e morte. A falta de integração dos $\mathrm{CP}$ aos protocolos de triagem, durante a pandemia, ignora as necessidades de pacientes que necessitam desse tipo de cuidado, sob a égide da falsa dicotomia de que a opção de não ofertar CP deve-se à necessidade de se fornecer tratamento (mesmo que agressivo) visando salvar a vida do paciente. Se as equipes de saúde optarem pelo último (tratamento agressivo em vez de exclusão), recursos escassos podem ser gastos em cuidados fúteis e onerosos para os pacientes que podem ser melhor atendidos por intervenções paliativas ${ }^{(2)}$.

Observa-se, a partir dos resultados de uma revisão sistemática ${ }^{(2)}$, fundamentada na análise de 95 publicações sobre prestação de CP em crises humanitárias, uma escassez de dados sobre as necessidades de $C P$ e intervenções fornecidas nesses contextos e a falta de consenso sobre a ética de fornecer ou limitar CP como parte da resposta à saúde humanitária. Tais resultados justificam a importância da realização de estudos sobre ética e CP em crises humanitárias, tal como a atual pandemia de COVID-19.

Para alguns pesquisadores ${ }^{(3)}$, o dever de cuidar é um componente crítico de qualquer plano de classificação de pandemia. Nesse sentido, o papel das equipes de CP é priorizar o alívio ao sofrimento, fundamental durante o curso da epidemia, em especial, no atendimento integral e contínuo daqueles pacientes não internados ou ambulatoriais. Deve-se incorporar ao planejamento de pandemias e desastres orientações éticas, para apoiar os provedores e profissionais de saúde que, de outra forma, podem tomar decisões precipitadas com a finalidade de atender o paciente urgente e assim ultrapassarem os limites éticos ${ }^{(4)}$.

Nesse contexto, o presente estudo objetiva analisar a percepção de profissionais de saúde sobre as questões bioéticas na tomada de decisão acerca dos recursos escassos no contexto dos CP durante a pandemia de COVID-19 no Brasil.

\section{MÉTODOS}

Estudo transversal, descritivo e de abordagem quantitativa, aprovado pelo Comitê de Ética em Pesquisa da instituição proponente, CAAE 31479820.5.0000.0021, parecer de $\mathrm{n}$ 4.042.275, atendendo às recomendações da Resolução do Conselho Nacional de Saúde/Ministério da Saúde (CNS/MS) 466/2012, o qual faz parte de um estudo maior intitulado: Percepções de profissionais da saúde sobre alocação de recursos escassos na pandemia de COVID-19.

Profissionais da saúde envolvidos diretamente na assistência à saúde ou gestão, em todos os níveis de atenção, durante a pandemia de COVID-19 no Brasil, foram convidados a participar da pesquisa. Desconsideraram-se os profissionais de saúde que estavam em trabalho remoto em razão dos fatores de risco relacionados à pandemia.

O processo de obtenção da amostra foi não probabilístico, uma vez que os participantes foram convidados por amostragem em cadeia. Cada pesquisador do estudo escolheu, em sua rede de contatos, profissionais de saúde de diferentes estados do Brasil. Essas pessoas foram chamadas de influenciadores, cujo objetivo foi compartilhar e estimular a participação na pesquisa. Os influenciadores enviaram o link da pesquisa aos profissionais das suas redes sociais e assim atingiuse uma diversidade de respondentes. Buscou-se obter uma amostra de abrangência nacional. As informações sobre o estudo também foram divulgadas mediante a comunicação social pela instituição proponente da pesquisa.

A coleta de dados ocorreu, entre julho e outubro de 2020, por meio de um questionário online autopreenchido pelos participantes, enviado por meios de comunicação digital e redes sociais, utilizando-se a ferramenta de formulários Google. 
Em junho de 2020, o questionário foi submetido a uma avaliação de conteúdo por cinco profissionais de saúde com diferentes formações, a fim de avaliar a clareza dos itens quanto à redação e à compreensão. O questionário sofreu adaptações após essa etapa. Antes de iniciar o preenchimento do formulário, aplicou-se o Termo de Consentimento Livre e Esclarecido (TCLE), por meio da mesma ferramenta, sendo disponibilizada sua cópia em PDF assinada pelo pesquisador responsável.

A primeira parte foi composta por um questionário contendo questões fechadas. Tratase de um instrumento construído pelos próprios pesquisadores, composto por quatro afirmações relacionadas à tomada de decisão, envolvendo alocação de recursos escassos em meio à pandemia de COVID-19, que o respondente deveria assinalar de acordo com sua percepção (Quadro 1).

As afirmativas foram redigidas e analisou-se a sua concordância ou não, a partir de dados encontrados na literatura ${ }^{(3-8)}$, a fim de avaliar as respostas dos participantes e responder ao objetivo da pesquisa, quanto à sua percepção, no processo de tomada de decisão perante as situações hipotéticas apresentadas. E, para uma melhor compreensão dos dados coletados, as questões foram sistematizadas em quatro eixos temáticos, ou seja, cada questão correspondeu a um eixo temático que subsidiou a análise do estudo: Restrição do acesso às Unidades de Terapia Intensiva (UTIs) ${ }^{(5-7)}$; Disponibilização de ventiladores $^{(4,6)}$; Decisão de triagem para recursos escassos $^{(5,7)}$; e Interrupção do atendimento a pacientes crônicos e paliativos na pandemia de COVID-19(3,8) (Quadro 1).

A pontuação total do questionário foi de 20 pontos, correspondendo a cinco pontos para cada resposta considerada correta e zero ponto para as respostas consideradas incorretas ou caso o participante escolhesse pela opção "não tenho condições de opinar". Quanto maior a pontuação, maior é o conhecimento dos critérios éticos na tomada de decisão sobre recursos escassos durante a pandemia de COVID-19. E, ao término do preenchimento, o participante recebia um feedback dos resultados.

Quadro 1 - Questões que compuseram a análise do estudo sobre dilemas em bioética, quanto à tomada de decisão envolvendo alocação de recursos escassos em meio à pandemia de COVID-19.

\begin{tabular}{|c|c|c|c|}
\hline Eixo temático 1. Restrição do acesso às Unidades de Terapia Intensiva (UTIs) & Concordo & Discordo & $\begin{array}{l}\text { Não tenho } \\
\text { condições } \\
\text { de opinar }\end{array}$ \\
\hline \multicolumn{4}{|l|}{$\begin{array}{l}\text { Afirmativa 1. Pode ser recomendável excluir o acesso às Unidades de Terapia Intensiva } \\
\text { (UTIs) a pacientes com prognóstico desfavorável, em meio à necessidade de destinação de } \\
\text { leitos para o atendimento a pessoas com melhor prognóstico, durante a pandemia de } \\
\text { COVID-19. }\end{array}$} \\
\hline Eixo temático 2. Disponibilização de ventiladores & Concordo & Discordo & $\begin{array}{l}\text { Não tenho } \\
\text { condições } \\
\text { de opinar }\end{array}$ \\
\hline \multicolumn{4}{|l|}{$\begin{array}{l}\text { Afirmativa } 2 \text {. Na disponibilização de ventiladores, é sempre recomendado priorizar aqueles } \\
\text { pacientes críticos com maior probabilidade de sobreviver até a alta hospitalar com o } \\
\text { tratamento. }\end{array}$} \\
\hline Eixo temático 3. Decisão de triagem para recursos escassos & Concordo & Discordo & $\begin{array}{l}\text { Não tenho } \\
\text { condições } \\
\text { de opinar }\end{array}$ \\
\hline \multicolumn{4}{|l|}{$\begin{array}{l}\text { Afirmativa } 3 \text {. Em caso de pacientes com prognóstico semelhante e com a mesma } \\
\text { gravidade/urgência de atendimento, é mais adequado destinar recursos por sorteio em vez } \\
\text { de ordem de chegada. }\end{array}$} \\
\hline $\begin{array}{l}\text { Eixo temático 4. Interrupção do atendimento a pacientes crônicos e paliativos na pandemia } \\
\text { de COVID-19 }\end{array}$ & Concordo & Discordo & $\begin{array}{l}\text { Não tenho } \\
\text { condições } \\
\text { de opinar }\end{array}$ \\
\hline $\begin{array}{l}\text { Afirmativa 4. No contexto de pandemia COVID-19, é adequado interromper o atendimento } \\
\text { a pacientes com necessidades urgentes, como câncer, diabetes, doenças cardíacas e } \\
\text { gestantes, visando à redução da circulação de pessoas em instituições de saúde. }\end{array}$ & & & \\
\hline
\end{tabular}

Fonte: elaborado pelos autores.

Posteriormente, os participantes preencheram itens referentes à profissão, grau de formação, estado de atuação profissional e perguntas sobre o seu conhecimento em bioética, tais como ter ou não ter conhecimento sobre bioética, como adquiriu o conhecimento, como 
considera o seu nível de conhecimento em relação à bioética, a importância da bioética na atuação profissional e a importância da bioética na elaboração de políticas públicas em um contexto de pandemia.

Os dados foram tabulados no programa

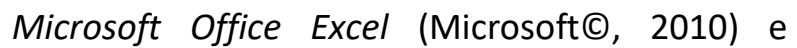
apresentados por meio de estatística descritiva. Realizaram-se análises de estatística descritiva, para a obtenção de média, desvio- padrão, frequências absolutas e relativas, por meio do software Statistical Package for Social Sciences (SPSS), versão 25.0.

\section{RESULTADOS E DISCUSSÃO}

Participaram 190 profissionais de saúde, e a sua caracterização encontra-se descrita na Tabela 1.

Tabela 1 - Caracterização dos profissionais da saúde atuantes durante a pandemia de COVID-19, Brasil, 2020 $\underline{(n=190) \text {. }}$

\begin{tabular}{|c|c|c|}
\hline & & \\
\hline & $\mathbf{N}$ & $\%$ \\
\hline \multicolumn{3}{|l|}{ Profissão } \\
\hline Enfermeiro(a) & 86 & 45,3 \\
\hline Médico(a) & 45 & 23,7 \\
\hline Dentista & 20 & 10,5 \\
\hline Farmacêutico(a) & 10 & 5,3 \\
\hline Fisioterapeuta & 5 & 2,6 \\
\hline Outros profissionais de saúde & 24 & 12,6 \\
\hline \multicolumn{3}{|l|}{ Grau de formação } \\
\hline Especialização & 88 & 46,3 \\
\hline Graduação & 53 & 27,9 \\
\hline Mestrado & 25 & 13,2 \\
\hline Doutorado & 12 & 6,3 \\
\hline Pós-Doutorado & 4 & 2,1 \\
\hline Outros & 8 & 4,2 \\
\hline \multicolumn{3}{|l|}{ Estado de Atuação } \\
\hline Mato Grosso do Sul (MS) & 72 & 37,9 \\
\hline São Paulo (SP) & 64 & 33,7 \\
\hline Minas Gerais (MG) & 14 & 7,4 \\
\hline Paraná (PR) & 14 & 7,4 \\
\hline Piauí (PI) & 4 & 2,1 \\
\hline Espírito Santo (ES) & 3 & 1,6 \\
\hline Outros Estados & 19 & 10 \\
\hline
\end{tabular}

Fonte: Dados da pesquisa (2020).

Enfermeiros e médicos eram constituídos, em sua maioria, por profissionais participantes, visto que são, frequentemente, os mais envolvidos no processo de tomada de decisão em conflitos éticos e na triagem de pacientes relativa à alocação de leitos e ventiladores durante períodos de pandemia ${ }^{(7,9)}$.

Um estudo ${ }^{(10)}$ realizado com médicos, enfermeiros e técnicos de enfermagem, em uma UTI pediátrica em Porto Alegre/Brasil, com o objetivo de avaliar as percepções desses profissionais sobre a participação no processo de tomada de decisão referente à limitação de suporte de vida em pacientes pediátricos terminais, identificou que médicos e equipe de enfermagem percebem falta de voz no processo de tomada de decisão.
A maioria dos participantes apresentava grau de formação, em nível de especialização, além de mestrado, doutorado e pós-doutorado, o que demonstra a complexidade da temática do estudo, a qual envolve a tomada de decisão mediante recursos escassos durante a pandemia da COVID-19(11). Acredita-se que a participação limitada de profissionais com outros níveis de formação e categorias profissionais deve-se, possivelmente, ao desconforto com a temática abordada, tal como também constatado em outro estudo $^{(10)}$, ou à sua falta de conhecimento sobre o assunto.

A maior participação de profissionais dos estados de Mato Grosso do Sul e São Paulo justifica-se pelo procedimento de amostragem em cadeia, visto que eram os locais em que havia a 
maior rede de contatos dos pesquisadores da equipe do estudo.

Na Tabela 2, observa-se a percepção dos participantes, em relação ao nível de conhecimento e importância da formação, na área de Bioética, para a atuação durante a pandemia.

Tabela 2 - Percepção dos profissionais da saúde, quanto ao nível de conhecimento e importância da bioética, para atuação durante a pandemia de COVID-19, Brasil, 2020 ( $n=190)$.

\begin{tabular}{|c|c|c|}
\hline \multicolumn{3}{|c|}{ Percepção sobre conhecimento em Bioética } \\
\hline & $\mathbf{n}$ & $\%$ \\
\hline \multicolumn{3}{|l|}{ Tem conhecimento sobre Bioética? } \\
\hline Sim & 144 & 75,8 \\
\hline Não & 36 & 18,9 \\
\hline Não sei & 10 & 5,3 \\
\hline \multicolumn{3}{|l|}{ Como adquiriu conhecimento em Bioética? } \\
\hline Durante a formação na Graduação & 95 & 50 \\
\hline Curso de Pós-Graduação & 27 & 14,2 \\
\hline Em educação permanente em serviço & 26 & 13,7 \\
\hline Outros cursos ou eventos na temática & 15 & 7,9 \\
\hline Não tenho conhecimento prévio em Bioética & 27 & 14,2 \\
\hline \multicolumn{3}{|c|}{ Qual é o seu nível de conhecimento sobre Bioética? } \\
\hline Amplo conhecimento & 6 & 3,2 \\
\hline Conhecimento suficiente & 80 & 42,1 \\
\hline Conhecimento mínimo & 21 & 11 \\
\hline Pouco conhecimento & 71 & 37,4 \\
\hline Nenhum conhecimento & 12 & 6,3 \\
\hline \multicolumn{3}{|c|}{ A importância da Bioética para atuação profissional durante a pandemia de Covid-19 } \\
\hline Muito importante & 88 & 46,3 \\
\hline Importante & 81 & 42,6 \\
\hline Pouco importante & 4 & 2,1 \\
\hline Não é importante & 1 & 0,5 \\
\hline Não posso opinar & 16 & 8,4 \\
\hline \multicolumn{3}{|c|}{$\begin{array}{l}\text { A importância da Bioética para elaboração de políticas públicas acerca da distribuição de } \\
\text { recursos em saúde na pandemia de Covid-19 }\end{array}$} \\
\hline Muito importante & 127 & 66,8 \\
\hline Importante & 50 & 26,3 \\
\hline Pouco importante & 1 & 0,5 \\
\hline Não é importante & 0 & 0 \\
\hline Não posso opinar & 12 & 6,3 \\
\hline
\end{tabular}

Fonte: Dados da pesquisa (2020).

A maioria declarou ter conhecimento suficiente sobre Bioética o qual foi adquirido na graduação, além de reconhecer a importância desse tipo de conhecimento, para a atuação e elaboração de políticas públicas, no que concerne à alocação de recursos escassos na pandemia de COVID-19.

O ensino de Bioética na graduação é essencial para a sensibilização dos futuros profissionais de saúde sobre as decisões envolvendo dilemas e problemas que virão a surgir durante a prática profissional| ${ }^{(12)}$. Alguns autores ${ }^{(13)}$ investigaram o conhecimento sobre Bioética, no processo de tomada de decisão, por discentes dos cursos de Fisioterapia, Farmácia, Enfermagem, Educação Física e Odontologia de uma universidade pública no estado da Bahia/Brasil. Os resultados sobre o conhecimento acerca do conceito de Bioética apontaram que pouco mais da metade $(52,4 \%)$ dos participantes apresentaram respostas satisfatórias, dentre os quais, os que cursavam Enfermagem, Fisioterapia e Odontologia obtiveram um percentual melhor de acertos, em relação às outras categorias pelo fato de contarem com disciplinas obrigatórias de Deontologia e/ou Bioética no currículo do curso.

A partir dos resultados de um estudo(14), realizado com profissionais da Estratégia de Saúde da Família (ESF), com o objetivo de investigar a percepção dos profissionais quanto à importância da ética e da bioética para as práticas em saúde, obteve-se que $54,8 \%$ não participaram de nenhuma atividade de treinamento sobre a temática, $16,1 \%$ dos participantes declararam ter adquirido conhecimento sobre bioética, durante a graduação ou por meio de cursos fornecidos pelo 
conselho profissional, 9,7\% com cursos ou capacitações promovidos por um órgão público, $6,5 \%$ adquiriram conhecimento, porém não especificaram onde e $3,2 \%$, em cursos de estudos técnicos, e a maioria $(83,9 \%)$ manifestou o desejo em aprender mais sobre a temática, reconhecendo sua importância.
$\mathrm{Na}$ Tabela 3, encontram-se descritos os percentuais de respostas corretas e incorretas dos profissionais de saúde participantes, no que tange às afirmativas apresentadas quanto à tomada de decisão perante dilemas bioéticos, envolvendo a alocação de recursos escassos, durante a pandemia de COVID-19, considerando-se as questões voltadas aos $\mathrm{CP}$.

Tabela 3 - Percepção dos profissionais da saúde, quanto ao nível de conhecimento e importância da bioética, para atuação durante a pandemia de COVID-19 Brasil, 2020 ( $n=190)$.

\begin{tabular}{|c|c|c|c|c|c|c|c|c|}
\hline \multirow[t]{2}{*}{ Eixos temáticos } & \multirow[b]{2}{*}{ Média } & \multirow[b]{2}{*}{ DP* } & \multicolumn{2}{|c|}{$\begin{array}{l}\text { Respostas } \\
\text { Corretas }\end{array}$} & \multicolumn{2}{|c|}{$\begin{array}{l}\text { Respostas } \\
\text { Incorretas }\end{array}$} & \multicolumn{2}{|c|}{$\begin{array}{l}\text { Não tenho } \\
\text { condições de } \\
\text { opinar }\end{array}$} \\
\hline & & & n & $\%$ & n & $\%$ & $\mathbf{N}$ & $\%$ \\
\hline $\begin{array}{l}\text { 1- Restrição do acesso às Unidades de Terapia Intensiva } \\
\text { (UTIs) }\end{array}$ & 4,29 & 6,163 & 75 & 39,5 & 93 & 48,9 & 22 & 11,6 \\
\hline 2- Disponibilização de ventiladores & 3,11 & 5,452 & 58 & 30,5 & 117 & 61,6 & 15 & 7,9 \\
\hline 3- Decisão de triagem para recursos escassos & 2,74 & 6,657 & 8 & 4,2 & 158 & 83,2 & 24 & 12,6 \\
\hline $\begin{array}{l}\text { 4-Interrupção do atendimento a pacientes crônicos e } \\
\text { paliativos na pandemia de Covid-19 }\end{array}$ & 4,87 & 1,954 & 177 & 93,2 & 11 & 5,8 & 2 & 1,1 \\
\hline
\end{tabular}

Fonte: Dados da pesquisa (2020).

*DP: Desvio-padrão.

Os achados deste estudo remetem ao conhecimento insuficiente dos profissionais da saúde, em relação aos dilemas éticos da prática profissional, envolvendo a alocação de recursos escassos na pandemia e que podem ameaçar o sucesso da resposta a uma emergência de saúde pública ${ }^{(6)}$. Tal insuficiência contradiz a percepção relatada pelos profissionais de que teriam conhecimento suficiente sobre a temática. Uma hipótese, para o elevado índice de erros, deve-se ao contexto de pandemia ocasionada por um patógeno novo, com cenário clínicoepidemiológico em constante mutação, em que a incerteza se impõe. Observa-se que o cérebro humano apresenta dificuldades para analisar conscientemente situações com diversas variáveis envolvidas ${ }^{(15-16)}$, contudo deve-se evitar um processo decisório, baseado na intuição e na insegurança, mesmo reconhecendo-se a importância da tomada de decisão rápida em um contexto emergencial ${ }^{(17)}$.

Outra hipótese relaciona-se à insuficiência da formação em Bioética, mediante a complexidade das reflexões bioéticas necessárias para a atuação profissional. Nota-se que muitos dos profissionais possuem certa dificuldade em identificar situações e problemas de natureza bioética no cotidiano, evidenciando-se uma confusão quanto a conceitos envolvendo a temática, ou então, acreditam que 0 conhecimento em Bioética esteja restrito ao campo das ciências da saúde ${ }^{(14)}$.
Os resultados acerca das percepções dos profissionais foram discutidos segundo os eixos a seguir.

\section{Restrição do acesso às Unidades de Terapia Intensiva (UTIs)}

A questão trazia a afirmativa de que "pode ser recomendável excluir o acesso às UTIs a pacientes com prognóstico desfavorável, em meio à necessidade de destinação de leitos, para o atendimento a pessoas com melhor prognóstico, durante a pandemia de COVID-19", sendo que (93 - 48,9\%) acreditam que, mesmo com prognóstico desfavorável e com a escassez de recursos, devese manter o acesso a UTI por pacientes com indicação de CP.

As Recomendações da Associação de Medicina Intensiva Brasileira (AMIB), para a abordagem da COVID-19, em medicina intensiva $^{(7)}$, em seus princípios de triagem, são de "A decisão de limitar o acesso a recursos escassos, como leitos de UTI, deve ser compartilhada e coordenada em conjunto com o diretor técnico do hospital e as autoridades de saúde em nível local, regional ou nacional. Essa decisão somente poderá ser tomada, após o esgotamento de recursos de cuidados críticos, em nível de sistema de saúde e com declaração de situação de catástrofe" ${ }^{\prime 7)}$. Além disso, dispõe que a avaliação tenha por base os níveis de prioridade, estabelecidos na Resolução CFM 2156/2016, em prioridade 1 a 5 . 
A Resolução CFM 2156/2016 estabelece cinco níveis de prioridade de acesso a UTIs: "Prioridade 1: pacientes que necessitam de intervenções de suporte à vida, com alta probabilidade de recuperação e sem nenhuma limitação de suporte terapêutico; Prioridade 2: pacientes que necessitam de monitorização intensiva, pelo alto risco de precisarem de intervenção imediata e sem nenhuma limitação de suporte terapêutico; Prioridade 3: pacientes que necessitam de intervenções de suporte à vida, com baixa probabilidade de recuperação ou com limitação de intervenção terapêutica; Prioridade 4: pacientes que necessitam de monitorização intensiva pelo alto risco de precisarem de intervenção imediata, mas com limitação de intervenção terapêutica; Prioridade 5: pacientes com doença em fase de terminalidade ou moribundos, sem possibilidade de recuperação". Em geral, esses pacientes não são apropriados para admissão na UTI (exceto se forem potenciais doadores de órgãos). No entanto seu ingresso pode ser justificado, em caráter excepcional, considerando as peculiaridades do caso e condicionado ao critério do médico intensivista ${ }^{(18)}$.

$O$ documento da AMIB ainda destaca que

"Os pacientes que pela alocação de prioridade não serão atendidos por cuidados de Medicina Intensiva deverão ser atendidos em outras unidades, com ênfase em controle de sintomas. Os cuidados, ainda que limitados, devem ser prestados de forma compassiva, de forma que os pacientes não se sintam abandonados"(7).

Sendo assim, a restrição à oferta de recursos escassos não deve implicar a descontinuidade da assistência em saúde, em suas outras dimensões, incluindo cuidados de final de vida se a morte for inevitável. Entretanto a falta de critérios para a restrição de acesso remete ao mau uso de leitos de UTI e contribui para a acentuação da condição de esgotamento desses recursos ${ }^{(7)}$. 0 Código de Ética Médica, Resolução CFM no 1.805 de 28 de novembro de 2006, afirma que, em "situações clínicas irreversíveis e terminais, o médico evitará a realização de procedimentos diagnósticos e terapêuticos desnecessários e propiciará aos pacientes sob sua atenção todos os cuidados paliativos apropriados" (Capítulo 1, inciso XXII) ${ }^{(19)}$.

Assim, a restrição de acesso a leitos de UTI por pacientes em CP não implica abandono ou discriminação desse tipo de paciente, mas, sim, no respeito à dignidade inerente a cada indivíduo que se aproxima do final da vida, proporcionando-lhe melhor qualidade de sobrevida, incluindo tratamentos adequados de controle de sintomas, em especial, controle da dor e acolhimento das suas necessidades emocionais, sociais e espirituais. ${ }^{(20)}$.

Neste eixo, observou-se que quase metade dos respondentes não concordou em restringir acesso a recursos (leitos), a fim de maximizar os benefícios para um maior número de pessoas. Acredita-se que tal discordância deve-se à percepção de que esse tipo de decisão deve considerar a opinião do paciente e família, respeitando-se o princípio da autonomia. Entretanto, em um contexto de pandemia como o da COVID-19, com escassez de leitos e ventiladores, médicos e profissionais de saúde têm a obrigação ética de proporcionar benefícios ao maior número de pessoas em detrimento às necessidades e percepções individuais de pacientes $^{(6)}$.

A aplicação do princípio da justiça deve ocorrer de maneira uniforme a todas as pessoas, devendo-se desvincular o critério de idade cronológica como única estratégia e incorporando-se outras variáveis como o grau de fragilidade, a idade biológica, bem como os valores e preferências dos pacientes de modo que a tomada de decisão ocorra de forma compartilhada entre a equipe e, sempre que possível, com o paciente e a família por meio de uma comunicação respeitosa, transparente e confiante. De modo geral, propõe-se a aplicação do princípio da justiça distributiva, priorizando-se o melhor custo/oportunidade e o princípio da proporcionalidade, eliminando-se condições em que se espera um benefício mínimo ${ }^{(21)}$.

Assim, o conhecimento técnico deve ser instrumentalizado, mediante a complexidade das circunstâncias envolvidas, para não admitir um olhar superficial e sem embasamento, regido por protocolos, visto que, nesses momentos as emoções influenciam o discernimento do indivíduo. Mesmo que a bioética considere pouco provável encontrar soluções consensuais para os conflitos morais persistentes na assistência à saúde, devem-se buscar soluções que sejam razoáveis e prudentes ${ }^{(11)}$.

Consequentemente, as instituições e clínicas, em todo o país, precisaram desenvolver protocolos para determinar métodos justos, sistemáticos e baseados em evidências, para decidir quem receberá os recursos de saúde, quando a demanda exceder a oferta disponível ${ }^{(8)}$.

\section{Disponibilização de ventiladores}


Neste eixo, apresentou-se aos participantes a seguinte afirmação: “Na disponibilização de ventiladores é sempre recomendado priorizar aqueles pacientes críticos com maior probabilidade de sobreviver até a alta hospitalar com o tratamento". Esperava-se que os participantes respondessem que não é 'sempre' recomendado, porém $61,6 \%$ (117) das respostas foram incorretas, pois concordaram com a afirmativa. Embora esse critério possa de imediato parecer adequado, por ter o propósito de fazer o maior bem para o maior número de pessoas, pode ser inadequado em alguns casos por ignorar outras considerações éticas, como a relevância de se considerar salvar o maior número de anos de vida (sobrevida em longo prazo) ${ }^{(4)}$.

Segundo a recomendação do U.S. Centers for Disease Control and Prevention ${ }^{(22)}$, a justificativa ética, para a restrição de acesso ou retirada do ventilador é que, em uma emergência de saúde pública, o objetivo de maximizar os resultados da população seria comprometido se os pacientes com pouca probabilidade de sobreviver tivessem permissão de usar indefinidamente os ventiladores. A realocação será angustiante, para os profissionais de saúde, pacientes e famílias, mas quando a ventilação mecânica é interrompida, é imprescindível um CP abrangente ${ }^{(6)}$.

No que quase parece um prenúncio, a distribuição de recursos limitados foi explorada recentemente por um intensivista do Departamento de Saúde Comunitária do Hospital Johns Hopkins nos Estados Unidos e sua equipe, em que participaram da pesquisa a comunidade, em geral e $72 \%$ dos participantes concordaram que existem certas pessoas ou grupos de pessoas que deveriam receber prioridade de tratamento em relação a outras, quando não há tratamento suficiente disponível para ajudar a todos. Eles também constataram que, em tempos de crise, os resultados em curto e longo prazo devem ser considerados, principalmente, na decisão de recursos escassos ${ }^{(23)}$.

A Organização Mundial da Saúde ${ }^{(20)}$ lançou, em 2018, um guia voltado aos CP, o qual busca auxiliar o profissional responsável pela triagem, no processo de integração do alívio de sintomas em tempos de crises e emergências humanitárias. Nesse instrumento, são categorizadas as recomendações de triagem, para pacientes em $\mathrm{CP}$, seguindo-se as seguintes classificações:

- Classificação vermelha: "Sobrevivência é possível com tratamento imediato", os CP devem ser integrados ao tratamento de manutenção da vida;

- Classificação azul: "Sobrevivência não é possível" em razão de cuidados disponíveis, assim os CP são para o controle de sintomas, acolhimento da família ou paciente e o luto;

- Classificação amarela: "Não está em imediato perigo de morte", mas o tratamento é necessário em breve. CP e/ou alívio dos sintomas podem ser necessários imediatamente;

- Classificação verde: "Necessitará de cuidados médicos em algum momento", nesse caso, após o tratamento de pacientes com condições mais críticas em $\mathrm{CP}$, pode ser necessário alívio dos sintomas e o acolhimento.

Sendo assim, visando salvar o maior número de anos de vida, ou seja, sobrevida em longo prazo, recomenda-se ${ }^{(7)}$ a utilização do instrumento Supportive and Palliative Care Indicators Tool (SPICT). Trata-se de uma ferramenta clínica utilizada pelos profissionais da saúde, para calcular indicadores clínicos, baseados em evidências das principais condições de saúde avançadas e progressivas, sem perspectiva terapêutica curativa, cuja expectativa de vida em longo prazo seja inferior a um ano por uma ou mais doenças para encaminhar o paciente aos CP. Esse instrumento permite uma revisão, na conduta e no tratamento vigente, proporcionando melhor planejamento do cuidado ${ }^{(24)}$.

\section{Decisão de triagem para recursos escassos}

A questão com o maior percentual de erro $(158-83,2 \%)$ foi a referente ao eixo temático que aborda a decisão de triagem em situações que envolvam recursos escassos. Neste cenário, apresentou-se a seguinte afirmativa: "Em caso de pacientes com prognóstico semelhante e com a mesma gravidade/urgência de atendimento, é mais adequado destinar recursos por sorteio em vez de ordem de chegada". Esperava-se que os participantes concordassem com essa afirmação, porém (158 - 83,2\%) não concordaram. Tal discordância, provavelmente, deveu-se à percepção de que a alocação por sorteio não seja a forma mais adequada do ponto de vista ético. Mas, a questão afirmava não que o sorteio seria o melhor critério, mas que seria mais adequado que considerar o critério "ordem de chegada".

Alguns autores ${ }^{(5)}$ recomendam que, em casos de empate clínico, é mais adequado o sorteio que a ordem de chegada, pois os 
tratamentos para a COVID-19 atendem a necessidades urgentes, o que significa que uma abordagem de "primeiro a chegar, primeiro a ser atendido" beneficiaria injustamente os pacientes que residissem mais próximo das unidades de saúde.

Com o objetivo de avaliar a percepção dos membros da comunidade sobre o processo de tomada de decisão ética sobre alocação de recursos médicos escassos, durante uma pandemia, pesquisadores ${ }^{(25)}$ conduziram uma investigação, no estado de Maryland nos Estados Unidos, cujos resultados demonstram que 0 critério "ordem de chegada", a priori, era bem aceito pela comunidade (82\%), porém, após a intervenção educativa sobre princípios éticos, realizada pelos pesquisadores, $52 \%$ desses participantes mudaram de opinião e passaram a acreditar que nunca se deve usar esse critério na triagem. Além disso, $80 \%$ manifestaram-se contra o critério sorteio após a intervenção. Portanto observa-se maior tendência na aceitação do critério ordem de chegada que sorteio. Do ponto de vista ético, o critério sorteio é mais adequado que a ordem de chegada, contudo a conduta mais adequada seria dispor-se de critérios vinculados a uma abordagem clínica individualizada, baseandose em orientações e protocolos que garantam a ética da saúde pública(25).

No contexto nacional, a AMIB, ABRAMEDE (Associação Brasileira de Medicina de Emergência), SBGG (Sociedade Brasileira de Geriatria e Gerontologia) e ANCP (Academia Nacional de Cuidados Paliativos) dispõem de um protocolo sobre a alocação de recursos escassos, durante a pandemia por COVID-19, que inclui um modelo de triagem que tem como objetivo propor uma ferramenta prática aos profissionais de saúde diante de decisões complexas associadas à alocação de leitos de UTI e ventiladores. Para dispor desse recurso, recomenda-se a utilização de instrumentos de avaliação individualizada de escores de gravidade, tais como o Sequential Organ Failure Assessment (SOFA) e o Acute Physiology And Chronic Health Evaluation (APACHE), sendo o SOFA mais utilizado. Esses instrumentos estratificam o grau de gravidade das disfunções orgânicas apresentadas por um paciente e, como critério de desempate, utilizamse, sequencialmente, escore de fragilidade clínica proposta pelo National Institute for Health and Care Excellence (NICE) no Reino Unido, a pontuação total do SOFA e, por último, a randomização, ou seja, o sorteio aleatório ${ }^{(7)}$.
Assim, para que o sistema de saúde funcione efetivamente, as equipes precisam saber quais decisões sobre a alocação recursos realmente possibilitarão salvar o maior número de vidas. Para isso, deve-se considerar a disponibilização desses recursos a pacientes não COVID-19 com quadro clínico instável, utilizandose os mesmos critérios. Isso porque esses pacientes precisarão de tratamento intensivo, para aliviar sua doença aguda, com certos limites terapêuticos, como não intubar. Além disso, protocolos de triagem mal projetados que interrompam atendimentos a pacientes crônicos ou que priorizem categorias de pessoas, para a retirada de suporte ventilatório, poderiam expor os sistemas de saúde a reivindicações éticas e legais de discriminação injusta ${ }^{(3)}$.

\section{Interrupção do atendimento a pacientes crônicos e paliativos na pandemia de COVID-19}

Neste eixo, apresentou-se a seguinte afirmação: "No contexto de pandemia COVID-19, é adequado interromper $o$ atendimento a pacientes com necessidades urgentes, como câncer, diabetes, doenças cardíacas e gestantes visando à redução da circulação de pessoas em instituições de saúde" e esperava-se que os respondentes discordassem, declarando não ser adequada essa interrupção. Essa questão envolveu o maior percentual de acertos (177$93,2 \%)$, e os participantes concordaram que a pandemia não justifica as interrupções no atendimento a condições que não a COVID-19 pelo sistema de saúde, pois, provavelmente, causaria mais mortes de pessoas com uma variedade de necessidades urgentes de saúde que de pacientes diagnosticados com COVID-19.

Em casos de crise, é o profissional responsável pelo tratamento desses pacientes que assume total responsabilidade de encorajálos a não interromper o tratamento, e não o próprio paciente ou amigo próximo, pois é o profissional de saúde que conhece o real impacto dessa interrupção. Nos $\mathrm{CP}$, o dever de cuidar é um componente crítico de qualquer plano de classificação de pandemia e desafiador, para as equipes de saúde, por remeter a conflitos relacionados a terapêuticas fúteis e prolongadoras do sofrimento ${ }^{(3)}$.

Deve-se destacar que, à medida que instituições e governos reduzam a prioridade de acesso a procedimentos eletivos e atendimento médico não urgente, eles devem estar atentos às necessidades usuais das regiões que atendem e 
reconhecerem que haverá pacientes enfermos que não têm COVID-19 e que precisam de atendimento especializado, tais como os pacientes em CP. Dispor desses recursos pode causar conflito entre o dever das instituições de cuidar dos pacientes e sua responsabilidade de administrar os recursos escassos ${ }^{(8)}$.

Assim, um bom planejamento de contingência pode ajudar a mitigar os efeitos da alocação e redistribuição de recursos. Além disso, os escores de gravidade da doença devem permitir a avaliação simultânea de pacientes com e sem COVID-19 e apoiar a alocação de recursos integrada, em vez de isolada ${ }^{(8)}$.

As limitações deste estudo incluem o número reduzido de participantes e a maior representação em dois estados brasileiros, contudo entende-se que os resultados desta pesquisa refletem a realidade no Brasil.

\section{CONCLUSÃO}

A percepção dos profissionais de saúde que estão atuando durante a pandemia de COVID-19 demonstra que, mesmo com conhecimento prévio sobre bioética no ensino de graduação, não se encontram suficientemente preparados para tomar decisões em situações dilemáticas na prática profissional. Torna-se necessário o incentivo à participação em programas de educação permanente em serviços voltados para temáticas de bioética.

Os profissionais também demonstraram conhecimento insuficiente na tomada de decisão diante dos problemas éticos relacionados aos CP em contextos de crise, tal como o da pandemia. Mostra-se urgente a necessidade de políticas públicas voltadas aos CP no país e políticas que considerem critérios éticos na alocação de recursos escassos em períodos de crise. Além disso, as instituições e serviços de saúde devem prever protocolos de triagem, capazes de reconhecer as necessidades dos pacientes, no contexto social de uma pandemia e com a participação de comitês de bioética.

Este estudo contribui para o conhecimento na área de Enfermagem, à medida que esses profissionais constituíram a maioria dos participantes, demonstrando a insuficiência do conhecimento e sua formação à tomada de decisão envolvendo a alocação de recursos escassos durante períodos de crise humanitária. A partir da identificação dos critérios éticos e instrumentos envolvidos na tomada de decisão, o enfermeiro poderá orientar suas ações e cuidados de modo mais assertivo e justo, garantindo-se uma assistência que considere uma visão ampliada e integral de saúde e que atenda ao princípio da equidade do SUS.

\section{REFERÊNCIAS}

1- Gostin LO, Friedman EA, Wetter SA. Responding to COVID-19: How to Navigate a Public Health Emergency Legally and Ethically. Hastings Cent. 2020;50(2):8-12. DOI: 10.1001/iama.2020.5046

2- Nouvet E, Sivaram M, Bezanson K, Krishnaraj G, Hunt $M$, Laat $L$, et al. Cuidados paliativos em crises humanitárias: Uma revisão da literatura. Ação Humanitária Int J. 2018;3(5). DOI: 10.1186/s41018-018-0033-8

3- Maglio I, Valdez P, Cámera L, Finn B, Klein M, Pincemim I, et al. Guías éticas para la atención durante la pandemia covid-19: recomendaciones multisocietarias para asignación de recursos medicina. Medicina 2020 [cited 2020 set 18]; 80(Suppl 3):45-64. Available in: https://www.medicinabuenosaires.com/indicesde-2020/volumen-80-ano-2020-s-3indice/guias eticas/

4- Biddison ELD, Gwon HS, Schoch-Spana M, Regenberger AC, Juliano C, Faden RR, et al. Scarce resource allocation during disasters: A mixedmethod community engagement study. Multicenter Study 2017;153(1):187-95. DOI: 10.1016/j.chest.2017.08.001

5- Emanuel EJ, Persad G, Hupshur R, Thomé B, Parker $M$, Glikman $M$, et al. Fair allocation of scarce medical resources in the time of Covid-19. N Engl J Med. 2020;382(21):2049-55. DOI: 10.1056/NEJMsb2005114

6- White DB, LO B. A framework for rationing ventilators and critical care beds during the COVID-19 pandemic. JAMA 2020;323(18):1773-4. DOI: 10.1001/jama.2020.5046

7- Kretzer L, Berbigier E, Lisboa R, Grumann AC, Andrade J. Recomendações da AMIB (Associação de Medicina Intensiva Brasileira), ABRAMEDE (Associação Brasileira de Medicina de Emergência, SBGG (Sociedade Brasileira de Geriatria e Gerontologia) e ANCP (Academia Nacional de Cuidados Paliativos) de alocação de recursos em esgotamento durante a pandemia por COVID-19. São Paulo: AMIB; 2020.

8- Laventhal N, Basak R, Dell ML, Diekema D, Elster N, Geis G, et al. The ethics of creating a resource allocation strategy during the COVID-19 pandemic. Pediatrics 2020;146(1):e20201243. DOI: $10.1542 /$ peds.2020-1243 
9- Conselho Federal de Enfermagem (COFEN). Recomendações gerais para organização dos serviços de saúde e preparo das equipes de enfermagem: As unidades de saúde devem se adequar às mudanças necessárias para enfrentamento da pandemia de COVID-19. Brasília: COFEN; 2020.

10- Trotta EA, Scarpa FC, Halal MG, Goldim JR, Carvalho PR. Health professionals' perceptions about the decision-making process in the care of pediatric patients. Rev Bras Ter Intensiva 2016;28(3):335-40. DOI: 10.5935/0103507X.20160057

11- Sanches MA, Cunha TR, Siqueira SS, Siqueira JE. Perspectivas bioéticas sobre tomada de decisão em tempos de pandemia. Rev Bioét. 2020;28(3):410-7. DOI: $10.1590 / 1983-$ $\underline{80422020283401}$

12- Finkler M, Verdi MIM, Caetano JC, Ramos FRS. Formação profissional ética: um compromisso a partir das diretrizes curriculares? Trab Educ Saúde 2010;8(3):449-62. DOI: $\quad$ 10.1590/S1981$\underline{77462010000300007}$

13- Biondo CS, Rosa RS, Ferraz MOA, Yarid SD. Perspectivas do conhecimento da bioética pelos acadêmicos de saúde para atuação profissional. Enfermería Actual de Costa Rica 2018;(35):63-74. DOI: 10.15517/revenf.v0i35.30014

14- Motta LCS, Vidal SV, Gomes AP, Lopes TCC, Rennó L, Miyadahira R, et al. En busca del ethos de la Estrategia Salud de la Familia: Una investigación bioética. Rev Bioét. 2015;23(2):360-72. DOI: 10.1590/1983-80422015232075

15- Gigerenzer G, Brighton H. Homo heuristicus: Why biased minds make better inferences. Top Cogn Sci. 2009;1(1):107-43. DOI: 10.1111/j.17568765.2008.01006.x

16- Han PKJ, Zikmund-Fisher BJ, Duarte CW, Knaus $M$, Black $A$, Scherer $A M$, et al. Communication of scientific uncertainty about a novel pandemic health threat: Ambiguity aversion and its mechanisms. J Health Commun. 2018;23(5):435-44. DOI: $10.1080 / 10810730.2018 .1461961$

17- Teixeira C, Rosa RG, Rodrigues Filho EM, Fernandes EO. The medical decision-making process in the time of the coronavirus pandemic. Rev Bras Ter Intensiva 2020;32(2):308-11. DOI: 10.5935/0103-507x.20200033

18- Conselho Federal de Medicina (CFM). Resolução CFM no 2.156, de 28 de outubro de 2016. Estabelece os critérios de admissão e alta em unidade de terapia intensiva. Diário Oficial da União 2016;1:138-9.
19- Conselho Federal de Medicina. Resolução CFM no 1.805 , de 28 de novembro de 2006. Na fase terminal de enfermidades graves e incuráveis é permitido ao médico limitar ou suspender procedimentos e tratamentos que prolonguem a vida do doente, garantindo-lhe os cuidados necessários para aliviar os sintomas que levam ao sofrimento, na perspectiva de uma assistência integral, respeitada a vontade do paciente ou de seu representante legal. Diário Oficial da União 2006;1:169.

20- World Health Organization (WHO). Integrating palliative care and symptom relief into the response to humanitarian emergencies and crises: A WHO guide. Geneva: World Health Organization; 2018.

21- Rubio O, Estella A, Cabre L, Saralegui-Reta I, Martin MC, Zapata L, et al. Recomendaciones éticas para la toma de decisiones difíciles en las unidades de cuidados intensivos ante la situación excepcional de crisis por la pandemia por covid19: Revisión rápida y consenso de expertos. Med Intensiva 2020;44(7):439-45. DOI: 10.1016/j.medin.2020.04.006

22- Centers for Disease Control and Prevention. Ethical Considerations for decision making regarding allocation of mechanical ventilators during a severe influenza pandemic or other public health emergency. USA: CDC; 2011.

23- Biddison ELD, Gwon HS, Schoch-Spana M, Cavalier R, White DB, Dawson $T$, et al. The community speaks: Understanding ethical values in allocation of scarce lifesaving resources during disasters. Ann Am Thorac Soc. 2014;11(5):777-83. DOI: 10.1513 / AnnalsATS.201310-3790C

24- Supportive and Palliative Care Indicator Tool (SPICT): SPICT-BRTM. 2020 [cited 2020 Nov 8]. Available https://www.spict.org.uk/thespict/spict-br/ 25- Romanò $M$. Fra cure intensive e cure palliative ai tempi di CoViD-19. Recenti Prog Med. 2020;111(4):223-30. DOI:10.1701/3347.33185

Nota: Artigo proveniente da Dissertação de Mestrado intitulada "Percepções de profissionais da saúde sobre alocação de recursos escassos na pandemia de COVID-19", do Programa de Pós Graduação Stricto Sensu em Enfermagem da Universidade Federal de Mato Grosso do Sul.

Recebido em: 18/10/2020

Aprovado em: 18/12/2020

Endereço de correspondência: 
Priscila Kelly da Silva Neto. Viela seis, no 62 - Setsul. CEP:

79610-370 - Três Lagoas/MS.

e-mail: priscila.baldonado@gmail.com 